\title{
Review
}

\section{An Overview of the Latest Developments in the Treatment of Feline Hyperthyroidism with Radioactive Iodine ${ }^{131}$ I}

\author{
Ferjan $\mathbf{P}^{1}$, Jeran $\mathbf{M}^{2,3, *}$ \\ 1. University of Ljubljana, Faculty of Veterinary Medicine, Ljubljana, Slovenia \\ 2. University of Ljubljana, Faculty of Health Sciences, Laboratory of Clinical Biophysics, Ljubljana, Slovenia \\ 3. University of Ljubljana, Faculty of Electrical Engineering, Laboratory of Physics, Ljubljana, Slovenia \\ * Correspondence: Marko Jeran; marko.jeran@fe.uni-lj.si
}

Citation: Ferjan P, Jeran M. An

overview of the latest developments in the treatment of feline hyperthyroidism with radioactive iodine ${ }^{131}$ I. Proceedings of Socratic Lectures. 2021; 6: 31-36.

https://doi.org/10.55295/PSL.2021.D. 005

\section{Publisher's Note: UL ZF stays} neutral with regard to jurisdictional claims in published maps and institutional affiliations.

\begin{abstract}
Feline hyperthyroidism is a disorder resulting from excessive concentrations of thyroid hormones in the systemic circulation. Parenteral application of radioiodine ${ }^{131} \mathrm{I}$ is the treatment of choice, but it can cause iatrogenic hypothyroidism. To minimalize that risk, algorithms for individual doses and prognosis factors have been suggested. Treatment of hyperthyroidism can worsen a pre-existing renal condition, therefore, renal function should always be assessed pre-and post-treatment. To make this treatment more available and widespread, oral administration of ${ }^{131} \mathrm{I}$ has been suggested. This contribution reviews the latest contributions on this subject and compares them to what is already known about the treatment of feline hyperthyroidism with radioactive iodine.
\end{abstract}

Keywords: endocrinology, feline hyperthyroidism, treatment, radioiodine ${ }^{131} \mathrm{I}$

\footnotetext{
Copyright: (c) 2021 by the authors. Submitted for possible open access publication under the terms and conditions of the Creative Commons Attribution (CC BY) license

(https://creativecommons.org/licens es/by/4.0/).
} 


\section{Introduction}

Hyperthyroidism is a disorder resulting from excessive concentrations of triiodothyronine (T3) and/or thyroxine (T4) in the systemic circulation. Thyroid hormones have a wide variety of actions, most important being the metabolism of carbohydrates, proteins, lipids, and increasing overall sympathetic drive (Mooney et al., 2012). An increase in their concentration causes a hypermetabolic state, responsible for symptoms, seen in hyperthyroidism (Graves et al., 2017).

\subsection{Etiology}

The cause of feline hyperthyroidism is, most commonly, benign adenomatous hyperplasia of one (30\%) or both $(70 \%)$ lobes (Mooney et al., 2012). Thyroid carcinoma is a rare occurrence (Graves et al., 2017), except when the initial benign hyperplasia is not treated - then it can potentially turn malignant (Carney et al., 2016). The underlying etiology is still not completely understood (Mooney et al., 2012). Multiple factors could be causing the abnormal growth of cells. In some hyperthyroid cats, mutations in thyroid-stimulating hormone (TSH) receptors and G-protein have been detected, leading to decreased ability of cAMP inhibition, resulting in excess secretion of T3 and T4. Some studies have shown that goitrogens (polybrominated diphenyl ethers, PBDEs) in food and/or the environment, isoflavones from soy, and varying concentrations of iodine in food might be some of the factors (Carney et al., 2016).

\subsection{Clinical manifestation}

Hyperthyroidism usually affects middle-aged and older cats. The most common clinical signs, associated with excessive production of thyroid hormones are weight loss with normal or increased appetite, tachycardia, systolic murmurs and gallop rhythm of the heart, intermittent gastrointestinal disorders (vomiting and/or diarrhea), polyuria/polydipsia, irritability, and palpable goiter (Mooney et al., 2012).

\subsection{Diagnosis}

$\mathrm{T} 4$ is the main secretory product of the feline thyroid gland, therefore the diagnostic method of choice is measuring serum concentrations of total thyroxine (tT4) (Graves et al., 2017). Most hyperthyroid cats have elevated tT4 concentrations, however, around $10 \%$ of all cases have serum total T4 concentrations within the reference interval. Especially in the early stages or mild cases, concentrations can fluctuate (Mooney et al., 2012). Concurrent diseases, such as diabetes mellitus, hepatic diseases, chronic kidney disease (CKD), congestive heart failure, etc., can also lower tT4 levels. Rechecking cats with symptoms of hyperthyroidism but no increase in tT4 is indicated. Tests for free T4 (fT4) have high sensitivity, but poor specificity and tend to be expensive. fT4 can be increased in cats with occult hyperthyroidism and can, as a result, be used in diagnosing euthyroid patients. Thyroid scintigraphy is an extremely sensitive test for diagnosing hyperthyroidism. Thyroid uptake of pertechnetate or radioactive iodine correlates strongly with hyperthyroxinemia, but the test is limited by its availability. Scintigraphy can also be in use for cats undergoing thyroidectomy - identifying unilateral versus bilateral disease and ectopic (Figure 1) (Graves et al., 2017). Other diagnostic tests include serum TSH measurement, T3 suppression test, TSH, and TRH stimulation test (Mooney et al., 2012; Graves et al., 2017).

\subsection{Treatment options}

Medical management with methimazole or carbimazole inhibits thyroid hormone synthesis and release, but it is non-curative. It is recommended for either pre-operative (to decrease metabolic and cardiac complications) or long-term management. Surgical thyroidectomy is considered to be very effective and a treatment of choice, but can lead to 
hypocalcemia if parathyroid glands are injured. Treatment with radioiodine 131I is usually considered the best treatment option for feline hyperthyroidism and will be the main topic of discussion in this contribution (Mooney et al., 2012). The research reviewed in this article outlines new approaches to the treatment that result in lower rates of iatrogenic hypothyroidism, thus making it safer. Alternative methods of ${ }^{131} \mathrm{I}$ administration are also suggested, possibly making the treatment more available. It also discusses how to deal with concurrent and post-treatment kidney damage.

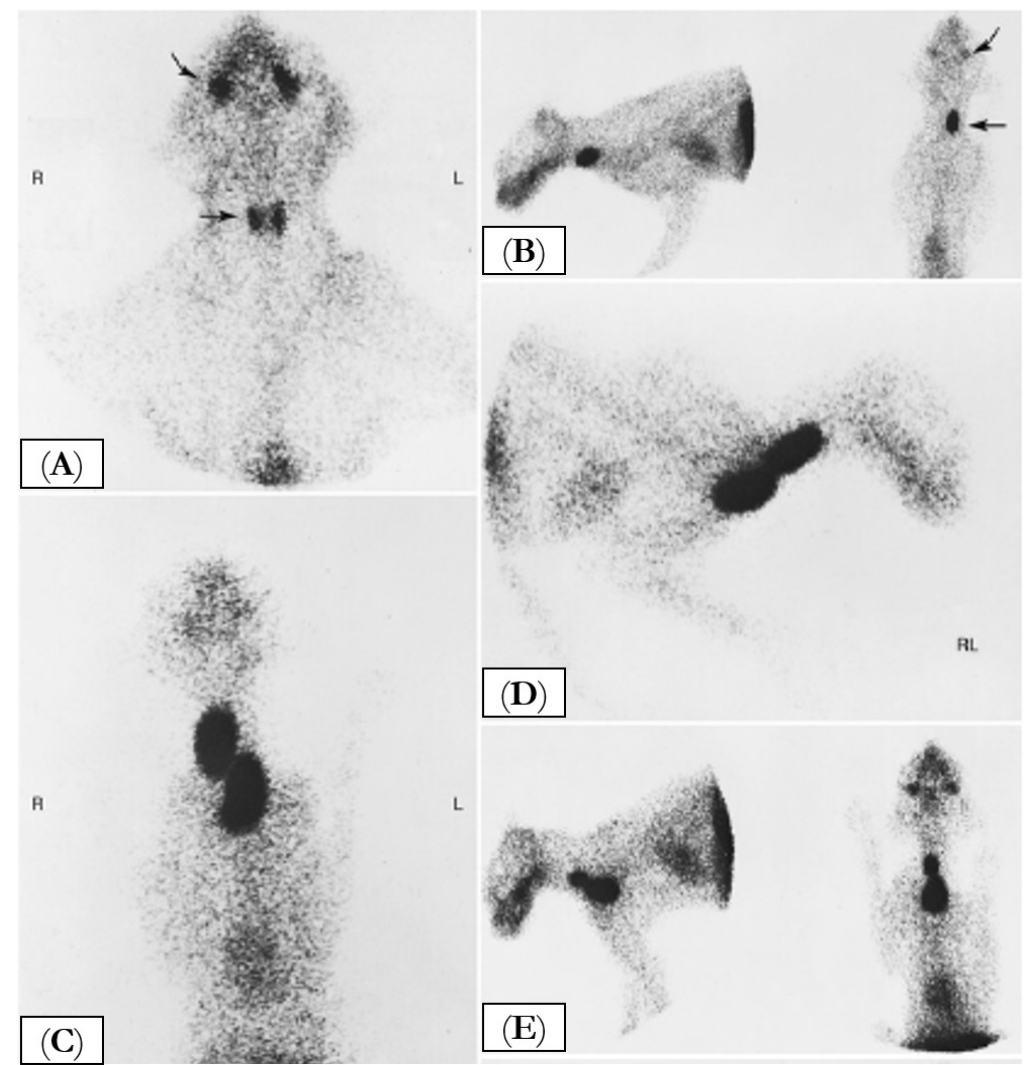

Figure 1. Thyroid scan (radioactive technetium - 99m) of a normal cat (A), thyroid scan of a cat with unilateral disease (B), thyroid scan of a cat with symmetric bilateral disease (C and D), thyroid scan of a cat with asymmetric bilateral disease (E). From Graves et al. (2017).

Thyroid hormones and thyroglobulin are the only iodinated organic molecules in the body, therefore any iodine, that comes into the body is taken to thyroid follicular cells. Radioiodine therapy exploits this process, using radioactive iodine isotope ${ }^{131} \mathrm{I}$, which concentrates in thyroid tissue (Mooney et al., 2012). Radioiodine emits beta particles, that cause tissue damage and can only travel up to $2 \mathrm{~mm}$, therefore are only locally destructive (Peterson et al., 2021a). It predominantly damages hyperactive cells (Graves et al., 2017). The main limitations of radioactive iodine treatment are the requirement for special licensure, isolation of cat from 3 days to 4 weeks after the treatment (Carney et al., 2016), and the possibility of developing hypothyroidism (Peterson et al., 2021a,b). T4 declines within the normal range in $4-12$ weeks post-treatment, and the symptoms can persist for a few months (Graves et al., 2017).

\section{Individualised dose and the prognosis factors}

The long-term prognosis for cats, treated with ${ }^{131} \mathrm{I}$ is good. Median survival time is estimated to be from 24 months -4 years. The most common causes of death in cats, treated with radioiodine are those normally associated with aging, such as malignancy and renal disease (Mooney et al., 2012). Euthyroidism in cats, treated with radioiodine, is not 
always achieved. $5 \%$ to $10 \%$ of cats remain persistently hyperthyroid and $20 \%$ to $50 \%$ develop iatrogenic hypothyroidism (Peterson et al., 2021b).

A recent study, conducted by Peterson et al. (2021a, b), aimed to individualize the dosing algorithm of ${ }^{131} \mathrm{I}$, and, as a result, minimize the risk of iatrogenic hypothyroidism (Peterson et al., 2021a). They also wanted to identify pretreatment factors, that could predict persistent hyperthyroidism and iatrogenic hypothyroidism after individualized treatment with 131I (Peterson et al. 2021b).

They found that with the fixed-dose $(3-5 \mathrm{mCi})$, iatrogenic hypothyroidism develops in $30-80 \%$ of cats within 6 months of treatments. By using an individualized algorithm, Paterson and Rishniw performed a prospective study that included 1400 hyperthyroid cats treated with ${ }^{131}$ (Peterson et al., $2021 \mathrm{a}, \mathrm{b}$ ). Cats that fitted the criteria, underwent an evaluation, that included a complete physical examination, routine laboratory testing (CBC, serum biochemical profile, complete urinalysis), determination of serum tT4, T3, and TSH concentrations, and qualitative and quantitative thyroid scintigraphy (Peterson et al., 2021b). Thyroid scintigraphy was performed by injecting sodium ${ }^{99 \mathrm{~m} T \mathrm{~T}}$ - pertechnetate (TcTU) intravenously, to classify the pattern of the disease (unilateral, bilateral, or multifocal disease) and to exclude thyroid carcinoma. They determined the volume of each thyroid tumor by calculating the percent thyroidal uptake of the injected TcTU (Peterson et al., 2021a).

Next, they assigned each of the parameters (T3 and T4 concentrations, TcTU, and thyroid volume) ${ }^{131}$ I dose scores. The higher the parameter value was, the higher the dose score. For example, serum $\mathrm{T}_{4}$ concentration of $15 \mu \mathrm{g} / \mathrm{dL}$ had a higher dose score than serum $\mathrm{T}_{4}$ concentration of $6 \mu \mathrm{g} / \mathrm{dL}$. This was done so there would be the same unit (mCi) for all of the parameters, which would simplify calculating the final dose (Peterson et al., 2021a).

The average ${ }^{131} \mathrm{I}$ dose score of all parameters combined was then calculated for each individual patient. To avoid overdosage, only $80 \%$ of this composite dose was used (Peterson et al., 2021a).

24 hours after administration, the percent of thyroidal ${ }^{131}$ I uptake was determined, and based on that, an additional ${ }^{131} \mathrm{I}$ dose was calculated and added to reach an adequate radiation dose $\left(200 \mu \mathrm{Ci}\right.$ per $\left.\mathrm{cm}^{3}\right)$ (Peterson et al., 2021a).

The average calculated final dose (dose, given on the first day plus the additional dose, given after 24 hours) was $1.9 \mathrm{mCi}$ (ranging from 1.0-10.6 mCi). Almost all cats (1380) required an additional ${ }^{131} \mathrm{I}$ administration on day 2 (Peterson et al., 2021a). Based on serum concentrations of T4 and TSH at 6 to 12 months after treatment, they divided cats into 4 categories: euthyroid (1047 cats $-74.8 \%$ ), overtly hypothyroid (57 cats $-4.1 \%$ ), subclinically hypothyroid ( 240 cats $-17.1 \%$ ), and persistently hyperthyroid (56 cats $-4 \%$ ) (Peterson et al., 2021a, b).

The study identified several pretreatment factors, that would help predict which cats would develop iatrogenic hypothyroidism or remain persistently hyperthyroid. Cats that developed ${ }^{131}$ I induced hypothyroidism were older, female, had a detectable TSH concentration, bilateral thyroid disease, milder severity, and a higher 24-hour percent ${ }^{131}$ I uptake. Cats, that were more likely to remain hyperthyroid tended to be younger and have higher severity and lower ${ }^{131}$ I uptake (Peterson et al., 2021b).

The study concludes that the calculated doses resulted in cure rates (percent of the cats that became euthyroid) similar or better to previously reported treatment rates but with much lower ${ }^{131} \mathrm{I}$ doses and that individualized doses of ${ }^{131} \mathrm{I}$ lower the prevalence of ${ }^{131} \mathrm{I}$ induced hypothyroidism (Peterson et al., 2021a).

If the patient becomes hypothyroiditic after the treatment, supplementation with levothyroxine might be needed, but it will also suppress pituitary TSH, which is needed to stimulate atrophied thyroid tissue. In those cases, it is of most importance to establish euthyroidism and avoid renal damage, while at the same time avoiding iatrogenic hypothyroidism (Carney, 2016). Monitoring of serum creatinine, tT4, and TSH is thus necessary (Carney, 2016). It was suggested that patients should be tested 30, 60, 90, and 180 days post-treatment (Carney, 2016). 


\section{3. ${ }^{131} \mathrm{I}$ administration}

131I is usually administered parenterally (Yu et al., 2020) - intravenously or subcutaneously (Graves et al., 2017). Oral iodine administration is also effective but is not often used, due to the possibility of vomiting after the administration, resulting in treatment failure. However, iodine for treatment $\mathrm{p} / \mathrm{o}$ is cheaper and easier to obtain. There is limited recent literature about the outcome of fixed oral doses for radioiodine in cats, and there is little known about the bioavailability of radioiodine. A retrospective study, that was published in 2019 by $\mathrm{Yu}$ et al. (2020) aimed to describe the percentage of hyperthyroid cats treated with a fixed dose of $3.7 \mathrm{mCi}$ radioiodine orally, that had tT4 concentrations within, below, or above the reference interval. The study included 227 cats, that had been treated using $3.7 \mathrm{mCi}$ radioiodine capsules orally. Maropitant citrate (Cerenia, Zoetis) was administered at $1 \mathrm{mg} / \mathrm{kg}$ subcutaneously, to minimize the risk of vomiting over the next 24 hours. Cats were fasted $12 \mathrm{~h}$ before the therapy, as an additional precaution. Out of 277 cats, 161 had tT4 concentrations available at diagnosis and after the treatment and were included in the study. Out of those, $133(82.6 \%)$ cats had tT4 concentrations within the reference interval, $4(2.5 \%)$ had tT4 levels above the reference interval and $24(14.9 \%)$ cats had tT4 concentrations below the reference interval. The study showed that the radioiodine dose should not necessarily be higher when it is administered orally (Yu et al., 2020). The dose used in this study was comparable or even lower than those usually used parenterally ( $\mathrm{Yu}$ et al., 2020). The rate of resolution of hyperthyroidism was comparable with other studies, as were the rates of persistent hyperthyroidism and iatrogenic hypothyroidism (Yu et al., 2020).

\section{Post-treatment renal insufficiency}

In cats with hyperthyroidism, high serum thyroid hormone concentrations result in increased renal blood flow. That is due to decreased vascular resistance and increased cardiac output, which lead to an increased glomerular filtration rate. Treatment of hyperthyroidism decreases glomerular filtration rate (GFR) (Stock et al., 2017), leading to chronic kidney disease in 15-60\% of cases in 6-8 months post-treatment (Graves TK, et al. 2017). Pre-treatment parameters could help predict post-treatment renal failure. Routinely used variables, such as serum urea and creatinine concentrations, urine specific gravity and proteinuria have low sensitivity and are influenced by cats hyperthyroid state (Stock et al., 2017). American College of Veterinary Internal Medicine (ACVIM) Panel recommends fully determining the renal status prior to hyperthyroidism treatment, using International Renal Interest Society (IRIS) staging guidelines, including determination of blood pressure, urine protein quantification, and body condition score. If azotemia develops post-treatment, IRIS Guidelines should be used for staging, treatment, and managing the disease (Carney et al. 2016). IRIS advises against leaving hyperthyroidism untreated to avoid kidney injury and an azotemic state (Carney et al. 2016). Elevated T4 can cause activation of the renin-angiotensin-aldosterone system, leading to increased cardiac output, volume overload, renal hypertension, ultimately progressing to CKD (Carney et al., 2016).

\section{Discussion}

Treatment with radioactive iodine ${ }^{131} \mathrm{I}$ is the most effective option of treatment for feline hyperthyroidism, resulting in a cure rate of $95 \%$ or higher (Carney et al, 2016). The general principle of treatment was to parenterally administer a fixed dose of ${ }^{131}{ }^{1}$, risking iatrogenic hypothyroidism. To avoid this complication, an estimation of a dose was used, which included severity of clinical disease, elevation in circulating tT4 concentration, and the size of goiter estimated by palpation (Graves et al. 2017). In 2021, a new algorithm was developed (Peterson et al, 2021a), that could be used to measure an individualized dose even more accurately, taking into account the concentration of thyroid hormones 
pre-treatment, the uptake of sodium ${ }^{99 \mathrm{~m} T c}$ - pertechnetate via thyroid scintigraphy, and thyroid volume. Lower doses resulted in lower incidents of iatrogenic hypothyroidism, while rates of euthyroidism stayed the same (Peterson et al, 2021a). Whilst successful, the downside might be the limited access to expensive nuclear equipment, used for thyroid scintigraphy and the radioiodine treatment itself.

A study by Peterson and Rishniw (2021b) has shown, that age, sex, TSH concentration, type of disease, and 24-hour percent ${ }^{131}$ I uptake are significant prognosis factors for iatrogenic hyperthyroidism. Older, female cats, that had detectable TSH concentration, bilateral thyroid disease, milder severity, and a higher 24 -hour percent ${ }^{131}$ I uptake, were more likely to be hypothyroid after the radioiodine treatment (Peterson et al., 2021b). It was reported (Stock et al, 2017) that the treatment for hyperthyroidism can worsen the preexisting renal disease, therefore kidney function should be assessed pre- and post-treatment, using IRIS staging guidelines. The use of methimazole before radioiodine treatment has been suggested for patients with a high risk of iatrogenic hypothyroidism and CKD since it is reversible and can in some ways predict the risk of significant renal compromise (Carney et al. 2016). Either way, hyperthyroidism should not be left untreated (Carney et al. 2016).

${ }^{131}$ I is usually administered parenterally, either intravenously or subcutaneously. Oral administration is cheaper and more widely available, but not usually used, due to the risk of vomiting, unpredictable resorption, and the concept, that a higher dose should be used (Yu et al., 2020). Recent studies (Yu et al., 2020) have shown, that when antiemetic agents are used, oral administration is successful with the same doses, that are used for parenteral treatment. Success rates were also comparable to those of parenteral treatment, but it remains unclear to what extent the resorption of ${ }^{131} \mathrm{I}$ is affected by the state of the gastrointestinal tract and its absorptive capacity. Those parameters are often changed in cats with hyperthyroidism, so further studies are needed to determine possible dose alterations and actual success rates.

Conflicts of Interest: The authors declare no conflict of interest.

\section{References}

1. Carney HC, Ward CR, Bailey SJ et al. 2016 AAFP Guidelines for the management of feline hyperthyroidism. J Feline Med Surg. 2016; 18: 400 - 416. DOI: 10.1177/1098612X16643252

2. Graves TK, Feline hyperthyroidism. In. Ettinger SJ, Feldman EC, Cote E, editors. Textbook of veterinary internal medicine. Elsevier, St. Louis, Missouri. 2017; pp. 4236 - 4255.

3. Mooney CT, Peterson M. Feline hyperthyroidism. In. Mooney CT, Peterson ME, editors. BSAVA manual of canine and feline endocrinology. BSAVA, Gloucester, United Kingdom. 2012; pp. 92 - 110. DOI: 10.22233/9781905319893.10

4. Peterson ME, Rishniw M. A dosing algorithm for individualized radioiodine treatment of cats with hyperthyroidism. J Vet Inetrn Med. Aug 2021a; 35: 2140 - 2151. DOI: 10.1111/jvim.16228

5. Peterson ME, Rishniw M. Predicting outcomes in hyperthyroid cats treated with radioiodine. J Vet Inetrn Med. 2021b: 1-10. DOI: 10.1111/jvim.16319

6. Stock E, Daminet D, Paepe D, et al. Evaluation of renal perfusion in hyperthyroid cats before and after radioiodine treatment. J Vet Inetrn Med. 2017; 31: 1658 - 1663. DOI: 10.1111/jvim.14852

7. Yu L, Lacorcia L, Finch S, Johnstone T. Assessment of treatment outcomes in hyperthyroid cats treated with an orally administered fixed dose of radioiodine. J Feline Med Surg. 2020; 22: 744 - 752. DOI: 10.1177/1098612X19884155 